\title{
Über den Umgang mit selektiv mutistischen Kindern Patienteninformation
}

Laura geht nun schon seit Wochen in den Kindergarten und noch immer hat sie kein Wort mit den Erzieherinnen oder den anderen Kindern gesprochen. Sie zeigt nur äußerst wenig Gestik oder Mimik und meidet weitestgehend Blickkontakt.

Lauras Eltern beschreiben sie als lebhaftes, lautes Kind, das zuhause eher Zeichen von Dominanz und Kontrolle als von Scheu an den Tag legt. Trotzdem bemerken auch sie Lauras verändertes Verhalten, wenn sie im Kindergarten oder unter anderen „fremden“ Menschen ist.

\section{Im Kindergarten}

$\nabla$

Oft sind es die Erzieherinnen im Kindergarten, die die Eltern erstmals mit dem veränderten Verhalten ihres Kindes konfrontieren. Das Schweigen der Kinder wird sich wahrscheinlich weder von selbst auflösen, noch von außen zu durchbrechen sein. Vielmehr sollte schrittweise eine Kommunikationsanbahnung über alternative Kommunikationswege gefunden werden. Schaffen Sie es, das Interesse des Kindes zu wecken, kann durch Malen, Rollenspiele, Pantomime oder eine gemeinsam erarbeitete Zeichensprache ein erster Austausch im Kindergarten stattfinden. Primär ist ein Kind mit Mutismus ein Kind. Es sollte an allen Aktivitäten beteiligt werden, ohne dabei in den Mittelpunkt gerückt zu werden. Helfen Sie den Eltern, einen geeigneten Therapieplatz für ihr Kind zu finden und stabilisieren Sie die nonverbalen Kommunikationsformen, bevor sie den nächsten Schritt einer verbalen Kontaktaufnahme angehen.

\section{In der Schule \\ $\nabla$}

Es ist wichtig, dass sowohl alle Lehrer als auch die Mitschüler ausreichend informiert sind, um dem Kind angemessene Hilfestellungen geben zu können und es so in die Klassengemeinschaft $\mathrm{zu}$ integrie- ren. Wie geht man mit den mündlichen Leistungen um, die mit bis zu 60\% die Gesamtnote mitbestimmen können? Aufgabe der Lehrer ist es, dem Kind Optionen zur Kompensierung seines Schweigens anzubieten und so seine Teilhabe am Unterricht zu gewährleisten. Hierbei kann das Schreiben einen kompensierenden Faktor darstellen. Es ist keine Alternative, das Kind seine Schulzeit „absitzen“ zu lassen, ohne es zu fordern. Fördern und Fordern stellen auch in dieser speziellen Situation die Grundpfeiler des Lernens dar. Durch sensibles, pädagogisches Handeln können Sie das Kind stärken, sodass es gegebenenfalls eigenständig den Mut zum Sprechen findet. Zeigen Sie dem Kind, dass es willkommen bei Ihnen ist und stärken Sie sein Selbstbewusstsein durch positive Verstärkung. Setzen Sie das Kind bezüglich seines Schweigens nicht unnötig unter Druck. Besprechen Sie sich mit seinem Therapeuten bezüglich des Vorgehens. Fängt das Kind an zu sprechen, nehmen Sie es als natürlichen Schritt hin und stellen sie das Kind und seine Stimme nicht in einen ungewollten Mittelpunkt. Ist das Schweigen bis zum Beginn der Schulzeit nicht durchbrochen, sollte spätestens zu diesem Zeitpunkt eine (schulbegleitende) Therapie begonnen werden.

\section{Therapien \\ $\nabla$}

Die großen Fachbereiche, in welchen Therapien angeboten werden, sind die Sprachtherapie, die Psychologie und die Psychiatrie. Die Relevanz der jeweiligen Therapieansätze hängt mit den individuellen Ursachen für das mutistische Verhalten des einzelnen Kindes zusammen.

Eine weitere, sich in Deutschland verbreitende Therapiemethode ist die Systemische Mutismus-Therapie (SYMUT), die speziell auf die Bedürfnisse und Symptome von Menschen mit Mutismus eingeht. Hier werden neben dem Phänomen des Nicht-Sprechens auch die typischen Ne-

\section{(Selektiver-) Mutismus}

(Selektiver-) Mutismus ist die „Sprechhemmung oder das Schweigen nach vollzogener Sprachentwicklung bei vorliegender Sprach- und Sprechfähigkeit“ [1]. Der Zeitpunkt des Schweigens kann vom Mutisten nicht bestimmt werden. Zumeist tritt es in den Situationen auf, in denen der Mutist durch den Einfluss ihm nicht ausreichend bekannter Menschen keine Kontrolle über das Geschehen erlangen kann.

benerscheinungen, wie eine symbiotische Mutter-Kind-Beziehung oder zwanghafte Kontrollmechanismen in die Therapie einbezogen.

\section{Fazit}

Kinder mit selektivem Mutismus sowie ihre Familien erwarten aufgrund des hohen Leidensdrucks viel von den verantwortlichen pädagogischen Einrichtungen. Für die Pädagogen heißt dies, die Kinder weder durch offensive Forderungen in den Rückzug zu treiben, noch den subjektiven Krankheitsgewinn durch die von den Kindern gewünschte Kontrolle zu groß werden zu lassen.

\section{Maria-Katharina Rolf, Frankfurt/Main}

Literatur und Literaturempfehlungen

1 Hartmann B, Lange M. Mutismus im Kindes-, Jugend- und Erwachsenenalter - Für Angehörige, Betroffene sowie therapeutische und pädagogische Berufe. 5.Aufl. Idstein: Schulz-Kirchner; 2010

- Rolf M-K. Väter und Mutismus - Eine bindungstheoretische Untersuchung. Saarbrücken: VDM-Verlag Dr.Müller; 2010.

überreicht durch Thieme und 